Revista ELectrónica de Investigación y EValuación Educativa
RELIEVE

ISSN: 1134-4032
e-Journal of Educational Research, Assessment and Evaluation

\title{
Perfiles de segregación escolar por nivel socioeconómico en España y sus Comunidades Autónomas
}

\author{
Profiles of school segregation by socioeconomic status in Spain and its Autonomous \\ Communities
}

Murillo, F. Javier \& Martínez Garrido, Cynthia

Universidad Autónoma de Madrid

\begin{abstract}
The research that estimate the magnitude of school segregation by socioeconomic status that has been done until now shown an overly simplistic image of the phenomenon that is not capable of reflecting his highly complex reality. This paper presents an alternative approach of analysis and interpretation of school segregation. Specifically, the aim of this research is to determine the profile of school segregation by socioeconomic status of Spain and its Autonomous Communities, and to establish models of segregation of the Communities. A special exploitation of the PISA 2015 data is conducted. We use the socioeconomic and cultural status of the families (NSEC) as a main variable. The analysis of the data is carried out in two phases: on the one hand, it is estimated the Gorard index using 19 minority groups; on the other hand, it is identified the segregation models and their characteristics using the analysis by clusters and k-means. The results show the existence of 17 different profiles of school segregation by socioeconomic status, one for each of the Autonomous Communities studied, and identify five behavior models of school segregation in Spain. This research shows how little is the knowledge about school segregation in Spain, and presents a more accurate alternative to estimate the magnitude of school segregation by socioeconomic status considering it, not as a point, but as a profile
\end{abstract}

Reception Date

2018 July 28

Approval Date 2019 February 03

Publication Date: 2019 February 05

Keywords: Segregation; School; Socio-economic status; Model; Spain

\begin{abstract}
Resumen
Las investigaciones que estiman la magnitud de la segregación escolar por nivel socioeconómico realizadas hasta el momento ofrecen una imagen excesivamente simplista que no es capaz de reflejar la realidad altamente compleja del fenómeno. Este trabajo presenta un enfoque alternativo de análisis e interpretación de la segregación escolar. Concretamente, busca determinar el perfil de segregación escolar por nivel socioeconómico de España y sus Comunidades Autónomas, y establecer modelos de segregación de las Comunidades. Para ello, se realiza una explotación especial de los datos de PISA 2015. Como variable criterio se utiliza el nivel socioeconómico y cultural de las familias (NSEC). El análisis de los datos se realiza en dos fases: de un lado, se estima el índice de Gorard utilizando 19 grupos minoritarios, de otro, se identifican modelos de segregación y sus características utilizando el análisis por conglomerados y de k-medias. Los resultados encontrados muestran la existencia de 17 perfiles diferentes de segregación escolar por nivel socioeconómico, uno para cada una de las Comunidades Autónomas estudiadas, e identifican cinco modelos de comportamiento de la segregación escolar por nivel socioeconómico en España. Esta investigación demuestra lo poco que se sabe sobre la segregación escolar en España y presenta una alternativa más precisa para estimar la magnitud de la segregación escolar por nivel socioeconómico considerándola, no como un punto, sino como un perfil
\end{abstract}

Palabras clave: Segregación; Escuela; Nivel socio-económico; Modelo; España

Fecha de recepción 2018 Julio 28

Fecha de aprobación 2019 Febrero 03

Fecha de publicación 2019 Febrero 05
Solo será posible tomar decisiones en política educativa pública que contribuyan a construir un mejor sistema educativo si se tienen informaciones actuales, fiables $\mathrm{y}$ precisas sobre su funcionamiento y desafíos. Y si hablamos de decisiones que afectan a las desigualdades en educación, que directamente inciden en la conformación de 
una sociedad más justa e inclusiva, la necesidad de datos y su mejor análisis será doble.

La segregación escolar, fenómeno por el cual los estudiantes se distribuyen desigualmente en unas escuelas $u$ otras en función de sus características personales o sociales, o de su condición, es uno de los elementos educativos que con mayor fuerza ataca a la igualdad de oportunidades educativas, y con ello a la consecución de una sociedad sin exclusiones. Sin embargo, aún se posee información insuficiente sobre la segregación escolar en general, y más en particular en España.

Efectivamente, las investigaciones que estiman la magnitud de la segregación escolar por nivel socioeconómico en España ofrecen una visión incompleta y demasiado simplista del fenómeno. Todos ellos resumen la información de la magnitud de la segregación en una o dos cifras, siendo la realidad mucho más compleja.

La investigación española que busca estimar la magnitud de la segregación escolar con datos estadísticamente representativos tiene poco más de diez años de existencia, siendo los trabajos de Ferrer, Ferrer y Castel (2006), de Benito y González (2007) y de Sánchez Hugalde (2007) pioneros en esta temática. Seguramente la razón haya que encontrarla en la necesidad de muestras estadísticamente representativas, aunque también del poco interés en estudiar este tema (Murillo, 2016). Efectivamente, es la disponibilidad de grandes bases de datos como la de la Generalitat de Cataluña (Benito \& González, 2007; Sánchez Hugalde, 2007) o, especialmente, de las diferentes ediciones del programa de evaluación internacional PISA, lo que ha contribuido al desarrollo de estos trabajos.

De esta manera, una amplia mayoría de las investigaciones que estiman la magnitud de la segregación escolar en España lo hacen para la Educación Secundaria. También hay trabajos españoles que estiman la segregación escolar en Educación Primaria, aunque en este caso no por segregación socioeconómica, sino por origen nacional de sus estudiantes (p. ej., Sánchez Hugalde, 2007; Síndic, 2008).

El uso de la base de datos de PISA hace que la mayoría de las investigaciones estimen la segregación para el conjunto del territorio estatal (Bonal, 2018; Ferrer, Ferrer \& Castel, 2006, 2008; Ferrer et al., 2011; MancebónTorrubia \& Pérez-Ximénez, 2009, 2010; Martínez y Ferrer, 2018; Murillo \& MartínezGarrido, 2018a, 2018b; Sicilia \& Simancas, 2018). En algunos casos, cuando los datos lo permiten, se analizan también algunas o todas las Comunidades Autónomas (Bonal, 2018; Ferrer et al., 2011; Mancebón-Torrubia \& Pérez-Ximénez, 2014; Martínez \& Ferrer, 2018; Murillo \& Martínez-Garrido, 2018a). Benito y González (2007), sin embargo, hacen una explotación especial de la base de datos de la Generalitat de Cataluña con lo que estiman la segregación en diferentes municipios. Mancebón-Torrubia y PérezXiménez (2014), por su parte recogen datos ad hoc de la Comunidad de Aragón, por lo que los resultados se circunscriben a esa Comunidad.

En la tabla 1 se recogen la decena de estudios más importantes que estiman la magnitud de la segregación escolar por nivel socioeconómico en España. Entre sus características diferenciadoras se encuentran:

1.Dimensión de segregación escolar. Dos son las dimensiones de segregación aplicables a la segregación escolar (St. John, 1966): la dimensión de uniformidad (Jahn, Schmid \& Schrag, 1947) por la que se entiende segregación escolar como la desigual distribución de los estudiantes en las escuelas, y la dimensión de exposición (Bell, 1954) que considera segregación escolar como la probabilidad de que un estudiante se encuentre en la escuela con alguien de su mismo grupo. Las diez investigaciones analizadas consideran la segregación escolar en su dimensión de uniformidad, y sólo dos abordan, además, la dimensión de exposición (Murillo y Martínez-Garrido, 2018a, 2018b).

2. Variable criterio. Las diferentes investigaciones utilizan diferentes 
variables para operativizar el nivel socioeconómico de las familias de los estudiantes. Para los trabajos que hacen explotaciones especiales de PISA, que son la gran mayoría, utilizan como variable criterio el nivel socioeconómico y cultural (ESCS) que la propia base de datos proporciona, variable continua (Bonal, 2018; Ferrer, Ferrer \& Castel, 2006; Ferrer et al., 2011; Mancebón-Torrubia \& PérezXiménez, 2010; Martínez \& Ferrer, 2018; Murillo \& Martínez-Garrido, 2018a, 2018b; Sicilia \& Simancas, 2018). Mancebón-Torrubia y Pérez-Ximénez, (2010), además, usan las variables categóricas "tipo de trabajo de los padres" y "nivel de estudios de los padres". Esos mismos autores, en el estudio sobre la segregación en Aragón (MancebónTorrubia \& Pérez-Ximénez, 2014) trabajan con la variable "ingresos familiares", también categórica. Benito y González (2007), por último, con datos de la Generalitat, usan la variable "capital instructivo de los padres”, categórica.

3. Índice de segregación utilizado. Existe una multiplicidad de índices para estimar la segregación, tanto que Gorard y Taylor (2002) han llegado a afirmar que había una "guerra de índices". Esta variabilidad tiene su fiel reflejo en la investigación española. Así, Ferrer, Ferrer y Castel (2006), Ferrer y colaboradores (2011), Murillo y MartínezGarrido (2018b) y Mancebón-Torrubia y Pérez-Ximénez (2009, 2010, 2014) utilizan el índice de Disimilitud (Duncan y Duncan, 1954). Murillo y MartínezGarrido (2018a, 201b) y Benito y González (2007) utilizan el índice de Gorard (2006). Murillo y Martínez-Garrido (2018b) estiman la segregación con el índice de Raíz Cuadrada (Hutchens, 2004). El índice de segregación de Coleman (Coleman, Hoffer \& Kilgore, 1982) es utilizado por Mancebón-Torrubia y Pérez-Ximénez (2010, 2014). Y, por último, Bonal (2018) y Sicilia y Simancas (2018) se decantan por utilizar el índice de Inclusión Social (OCDE, 2010).
4. Elección de grupo minoritario. El índice de Disimilitud, el de Gorard, el de Raíz Cuadrada y el de Coleman, es decir todos los usados por la investigación española con la excepción del índice de Inclusión Socioeconómica, exigen la existencia de un grupo mayoritario y otro minoritario para su estimación (Murillo, 2016). Los trabajos analizados con variables criterio categóricas han optado por diferentes caminos. Benito y González (2007) estudian la segregación para tres grupos minoritarios: estudiantes cuyos padres no tuvieran estudios, con estudios postobligatorios y con universitarios. Mancebón-Torrubia y Pérez-Ximénez (2009), por su parte, usa estudiantes con madres sin estudios o solo con estudios primarios, y con el tipo de trabajo de los padres blue collar (trabajadores que realizan tareas manuales y obreros en los sectores de la construcción, la industria, la agropecuaria, el mantenimiento de locales, el área metal-mecánica, las instalaciones y el mantenimiento técnico). Estos mismos autores, en su estudio de 2014 usan como variable que los ingresos familiares sean inferiores a 1.200 euros al mes (MancebónTorrubia \& Pérez-Ximénez, 2014). Sin embargo, cuando la variable criterio es continua es preciso dicotomizarla, y ahí radica la dificultad.

La mayoría de las investigaciones españolas que estiman la magnitud de la segregación escolar por nivel socioeconómico utilizan la variable nivel socioeconómico y cultural como criterio. Como dicha variable es continua es necesario determinar un punto de corte para dicotomizarla y determinar de esta manera el grupo mayoritario y el minoritario. La mayoría de los trabajos han optado por el $25 \%$ de los estudiantes con familias de menor nivel socioeconómico y cultural (cuartil 1, Q1), es el caso de Ferrer, Ferrer y Castel (2006), Ferrer y colaboradores (2011), Martínez y Ferrer (2018). Murillo y MartínezGarrido (2018b) añade el 25\% de los estudiantes con familias de mayor nivel socioeconómico y cultural (cuartil 4, Q4), y esos mismos autores en el otro trabajo de ese 
año (Murillo \& Martínez-Garrido, 2018a), consideran también el $10 \%$ de los estudiantes con familias de menor nivel socioeconómico (percentil 10, P10) y el 10\% con mayor (percentil 90, P90).

La decisión de elegir un grupo minoritario u otro no parece atenerse a ninguna razón objetiva. Es más, revisando otras investigaciones de carácter internacional se observan otras decisiones. Así, por ejemplo, Gasparini y otros (2011) y Jaume (2013) utilizan como punto de corte el percentil 20, Benavides, León y Etesse (2014) y Massey, Rothwell y Domina (2009) utilizan el primer cuartil, Valenzuela, Bellei y De los Ríos (2008) utilizan el percentil 30, Arenas y Hindriks (2017) y Vázquez (2012) utilizan la mediana, Valenzuela, Bellei y De los Ríos (2008) utilizan el percentil 70, y Krüguer (2014) y Murillo y Martínez-Garrido (2017) el cuarto cuartil.

La decisión de usar un grupo minoritario u otro tiene diferentes implicaciones. En primer lugar, que evidentemente, en función del punto de corte tomado los resultados de la magnitud de la segregación escolar son diferentes. Tal y como se observa en los resultados obtenidos por Murillo y MartínezGarrido (2008a), el valor de la segregación puede variar desde 0,20 a 0,45 en función del punto de corte utilizado (valor estimado a través del índice de Aislamiento para los individuos que conformar el percentil $10 \mathrm{y}$ el cuarto cuartil, respectivamente). Pero también que solo se tiene información de algunos grupos, desconociendo lo que sucede con otros.

Con todo ello, es posible afirmar que la investigación que estima la magnitud de la segregación escolar por nivel socioeconómico en España, y por extensión, en el resto de países, da una imagen simplista y parcial del fenómeno, pues sus resultados se reducen a un número que no es capaz de reflejar una realidad altamente compleja. Porque, en realidad, la segregación no es un número, es un perfil.

La presente investigación presenta un método de estimación de la segregación escolar por nivel socioeconómico preciso que permite solventar las deficiencias, la parcialidad y simplicidad de los estudios previos. Concretamente, esta investigación busca determinar el perfil de segregación escolar por nivel socioeconómico en Educación Secundaria Obligatoria en España y sus Comunidades Autónomas, y, a partir de ello, establecer diferentes modelos de segregación escolar existentes en el país.

(continúa en página siguiente) 
Murillo, F. Javier \& Martínez-Garrido, Cynthia (2019). Perfiles de segregación escolar por nivel socioeconómico en España y sus Comunidades Autónomas. RELIEVE, 25(1), art. 1. doi: http://doi.org/10.7203/relieve.25.1.12917

Tabla 1. Características de los 10 estudios españoles más importantes que estiman la magnitud de la segregación escolar por nivel socioeconómico y cultural

\begin{tabular}{|c|c|c|c|c|c|c|c|}
\hline Estudio & DiMENSIÓN & VARIABLE CRITERIo & $\begin{array}{l}\text { NIVEL EDUCATIVO } \\
\text { (BASE DATOS) }\end{array}$ & Población & ÍNDICE & GRUPO MINORITARIO & RESUltados * \\
\hline $\begin{array}{l}\text { Ferrer, Ferrer y Castel } \\
(2006)\end{array}$ & Uniformidad & $\begin{array}{l}\text { Nivel socioeconómico y } \\
\text { cultural }\end{array}$ & $\begin{array}{l}\text { Secundaria (PISA } \\
\text { 2003) }\end{array}$ & España y Cataluña & $\mathrm{D}$ & Q1 & D: 0,40 \\
\hline \multirow{3}{*}{ Benito y González (2007) } & \multirow{3}{*}{ Uniformidad } & \multirow{3}{*}{$\begin{array}{l}\text { Capital instructivo de los } \\
\text { padres }\end{array}$} & \multirow{3}{*}{$\begin{array}{l}\text { Secundaria (Datos } \\
\text { de la Generalitat de } \\
\text { Cataluña 2004) }\end{array}$} & \multirow{3}{*}{$\begin{array}{l}\text { Municipios } \\
\text { Cataluña }\end{array}$} & \multirow{3}{*}{ G } & Sin estudios & G: 0,45 \\
\hline & & & & & & $\begin{array}{c}\text { Estudios } \\
\text { postobligatorios } \\
\end{array}$ & G: 0,34 \\
\hline & & & & & & Estudios universitarios & G: 0,37 \\
\hline \multirow{3}{*}{$\begin{array}{l}\text { Mancebón-Torrubia y } \\
\text { Pérez-Ximénez (2009, 2010) }\end{array}$} & \multirow{3}{*}{ Uniformidad } & \multirow{3}{*}{$\begin{array}{l}\text { Nivel de estudios de los } \\
\text { padres } \\
\text { Tipo de trabajo de los padres } \\
\text { Nivel socioeconómico y } \\
\text { cultural }\end{array}$} & \multirow{3}{*}{$\begin{array}{l}\text { Secundaria (PISA } \\
\text { 2006) }\end{array}$} & \multirow{3}{*}{ España } & \multirow{3}{*}{$\mathrm{D}, \mathrm{C}$} & $\begin{array}{c}\text { Sin estudios o sólo } \\
\text { primarios }\end{array}$ & D: $0,29 \quad$ C: 0,06 \\
\hline & & & & & & Blue collar ** & D: $0,31 \quad$ C: 0,11 \\
\hline & & & & & & Q1 & D: 0,39 \\
\hline $\begin{array}{l}\text { Ferrer y colaboradores } \\
(2011)\end{array}$ & Uniformidad & $\begin{array}{l}\text { Nivel socioeconómico y } \\
\text { cultural }\end{array}$ & $\begin{array}{l}\text { Secundaria (PISA } \\
\text { 2009) }\end{array}$ & España y CCAA & $\mathrm{D}$ & Q1 & D: 0,38 \\
\hline $\begin{array}{l}\text { Mancebón-Torrubia y } \\
\text { Pérez-Ximénez (2014) }\end{array}$ & Uniformidad & Ingresos familiares & $\begin{array}{l}\text { Secundaria (Datos } \\
\text { propios 2005) }\end{array}$ & Aragón & $\mathrm{D}, \mathrm{C}$ & $\begin{array}{c}\text { Ingresos inferiores a } \\
1200 \text { eur/mes }\end{array}$ & D: $0,18 \quad$ C: 0,05 \\
\hline Bonal (2018) & Uniformidad & $\begin{array}{l}\text { Nivel socioeconómico y } \\
\text { cultural }\end{array}$ & $\begin{array}{l}\text { Secundaria (PISA } \\
\text { 2012) }\end{array}$ & España y Cataluña & IIS & No aplica & IIS: 0,75 \\
\hline Martínez y Ferrer (2018) & Uniformidad & $\begin{array}{l}\text { Nivel socioeconómico y } \\
\text { cultural }\end{array}$ & $\begin{array}{l}\text { Secundaria (PISA } \\
\text { 2006-2015) }\end{array}$ & $\begin{array}{l}\text { España, C. Madrid, } \\
\text { Cataluña, P. Vasco y } \\
\text { Andalucía }\end{array}$ & G & Q1 & G: 0,27-0,31 \\
\hline \multirow{2}{*}{$\begin{array}{l}\text { Murillo y Martínez-Garrido } \\
\text { (2018a) }\end{array}$} & Uniformidad & \multirow{2}{*}{$\begin{array}{l}\text { Nivel socioeconómico y } \\
\text { cultural }\end{array}$} & \multirow{2}{*}{$\begin{array}{l}\text { Secundaria (PISA } \\
\text { 2015) }\end{array}$} & \multirow{2}{*}{ España y CCAA } & G & P10, Q1, Q4, P90 & $\begin{array}{l}\text { P10-G: 0,41 Q1-G: 0,31 } \\
\text { Q4-G: 0,30 P90-G: 0,46 }\end{array}$ \\
\hline & Exposición & & & & A & P10, Q1, Q4, P90 & $\begin{array}{l}\text { P10-A: 0,20 Q1-A: 0,39 } \\
\text { Q4-A: 0,45 P90-A: 0,24 }\end{array}$ \\
\hline \multirow{3}{*}{$\begin{array}{l}\text { Murillo y Martínez-Garrido } \\
\text { (2018b) *** }\end{array}$} & \multirow{2}{*}{ Uniformidad } & \multirow{3}{*}{$\begin{array}{l}\text { Nivel socioeconómico y } \\
\text { cultural }\end{array}$} & \multirow{3}{*}{$\begin{array}{l}\text { Secundaria (PISA } \\
\text { 2000-2015) }\end{array}$} & \multirow{3}{*}{ España y CCAA } & \multirow{2}{*}{ G, D, H } & Q1 & G: $0,31 \quad$ D: $0,42 \quad H: 0,17$ \\
\hline & & & & & & Q4 & G: $0,35 \quad$ D: $0,47 \quad H: 0,20$ \\
\hline & Exposición & & & & A & Q1 & A: 0,39 \\
\hline & & & & & & Q4 & A: 0,45 \\
\hline Sicilia y Simancas (2018) & Uniformidad & cultural & 2015) & España y CCAA & IIS & No aplica & IIS: 0,72 \\
\hline
\end{tabular}

Nota: G: índice de Gorard. D: índice de Disimilitud. IIS: índice de Inclusión Social. H: índice de Raíz Cuadrada. C: índice de segregación de Coleman. A: índice de Aislamiento. (*) Cifras de la segregación escolar por nivel socioeconómico del conjunto de España, excepto en Benito y González (2007) que es el valor promedio de los municipios estudiados y en Mancebón-Torrubia y Pérez-Ximénez (2014), que es Aragón. ${ }^{\star *}$ La categoría profesional Blue collar hace referencia a los trabajadores que realizan tareas manuales y obreros en los sectores de la construcción, la industria, la agropecuaria, el mantenimiento de locales, el área metal-mecánica, las instalaciones y el mantenimiento técnico. (***) Solo resultados de 2015. 
Murillo, F. Javier \& Martínez-Garrido, Cynthia (2019). Perfiles de segregación escolar por nivel socioeconómico en España y sus Comunidades Autónomas. RELIEVE, 25(1), art. 1. doi: http://doi.org/10.7203/relieve.25.1.12917

\section{Método}

Para alcanzar dichos objetivos se lleva a cabo una explotación secundaria de los datos de España en la base de datos PISA 2015.

Como variable criterio se ha utilizado el nivel socioeconómico y cultural de las familias (NSEC). Este índice ha sido generado por PISA con base a la información aportada por los estudiantes y sus familias en los cuestionarios. El índice NSEC se obtiene a partir de las siguientes variables: el Índice Socioeconómico Internacional de la Situación Ocupacional de las familias (ISEI); y los índices construidos por PISA relativos al nivel más alto de educación alcanzado por los padres del estudiante convertido en años de escolaridad, el índice de nivel de riqueza familiar, el índice de nivel de recursos educativos disponibles para el estudiante en el hogar, y por último, el índice de nivel de posesiones culturales del hogar familiar. El valor de la variable NSEC se expresa como variable continua tipificada para cada país, con media 0 y desviación típica 1. El NSEC de cada país se calcula con la media de los índices de los estudiantes del país, considerando la ponderación dado que cada estudiante tiene un peso equivalente al número de individuos de la población a la que representa.

La muestra total se compone por 39.066 estudiantes de 1.177 escuelas. En PISA 2015, además de la muestra estatal (6.736 estudiantes de 201 escuelas), todas las Comunidades Autónomas han ampliado su muestra regional para poder recabar datos que sean comparables a nivel internacional, con un promedio de 1.800 estudiantes de 54 escuelas en cada Comunidad Autónoma, con la excepción de País Vasco que duplica dicha cifra. La tabla 2 muestra la distribución de estudiantes y escuelas de la muestra en cada Comunidad Autónoma.

Tabla 2. Muestra del estudio. Número de estudiantes y de centros

\begin{tabular}{lcc}
\hline & Estudiantes & Escuelas \\
\hline Andalucía & 1.813 & 54 \\
Aragón & 1.798 & 53 \\
Ppdo. Asturias & 1.790 & 54 \\
Illes Balears & 1.797 & 54 \\
Canarias & 1.842 & 54 \\
Cantabria & 1.924 & 56 \\
Castilla y León & 1.858 & 57 \\
Castilla-La Mancha & 1.889 & 55 \\
Cataluña & 1.769 & 52 \\
Extremadura & 1.809 & 53 \\
Galicia & 1.865 & 59 \\
La Rioja & 1.461 & 47 \\
C. de Madrid & 1.808 & 51 \\
Reg. de Murcia & 1.796 & 53 \\
C. F. Navarra & 1.874 & 52 \\
País Vasco & 3.612 & 119 \\
Com. Valenciana & 1.625 & 53 \\
\hline Total España & 6.736 & 201 \\
\hline
\end{tabular}

Fuente: Elaboración propia a partir de PISA 2015. 
Para dar respuesta a los objetivos de la investigación el análisis de los datos se realiza en dos fases. De un lado, para estimar el perfil de segregación escolar por nivel socioeconómico en las Comunidades Autónomas se utiliza el índice de segregación de Gorard. La propiedad de este índice de mantenerse invariable ante los cambios en la composición del grupo minoritario hace su uso recomendable frente a otros índices como el índice de Disimilitud (Gorard, 2006).

De acuerdo con el índice de Gorard, el nivel de segregación escolar se estima como la proporción de sujetos del grupo minoritario que deberían cambiar de unidad organizativa para que exista una distribución homogénea de los mismos entre los distintos establecimientos. Las estimaciones realizadas en la presente investigación se obtienen utilizando las ponderaciones finales aportadas por el estudio PISA para cada estudiante. La utilización de los datos ponderados compensa los posibles sesgos surgidos por el muestreo o de la no-respuesta por parte de las escuelas y los estudiantes; su empleo, además, permite derivar estimaciones apropiadas de los valores poblacionales. La ponderación implica reconocer que la importancia de las unidades de la muestra varía entre las mismas y que las más relevantes deben contribuir más que las restantes al cálculo de cualquier estimación poblacional. Así, una unidad con baja probabilidad de selección se considera más importante que una unidad con una alta probabilidad de selección. Los pesos son inversamente proporcionales a dicha probabilidad de selección (OCDE, 2016).

El procedimiento de cálculo del índice de Gorard es:

$$
\mathrm{G}=\frac{1}{2} \sum_{i=1}^{k}\left|\frac{X_{1 i}}{X_{1}}-\frac{T_{i}}{T}\right|
$$

Donde, para cada Comunidad Autónoma, $\mathrm{x}_{1 \mathrm{i}}$ representa el número de estudiantes del grupo minoritario en la escuela $i, \mathrm{X}_{1}$ es el número total de estudiantes del grupo minoritario en todas las escuelas de la Comunidad, $\mathrm{T}_{\mathrm{i}}$ es el número total de estudiantes en la escuela $i, \mathrm{y} \mathrm{T}$, número total de estudiantes en cada Comunidad.

Para definir el grupo minoritario se utiliza el punto de corte del $5 \%$ de manera que se crean 19 grupos minoritarios de la variable nivel socioeconómico y cultural de la familia de los estudiantes (variable continua). Estos grupos recogen desde el $5 \%$ de los estudiantes cuyas familias cuentan con menor nivel socioeconómico y cultural, hasta el $5 \%$ de estudiantes con mayor nivel.

Para alcanzar el segundo de los objetivos, identificar los diferentes modelos de segregación escolar entre las CCAA, se realiza un doble análisis de conglomerados: un análisis jerárquico para explorar le existencia de grupos o modelos tomando la distancia euclídea al cuadrado como medida de asociación y un análisis de K-medias para verificar sus características.

\section{Resultados}

\section{a) Perfil de segregación}

En la figura 1 se ha representado el perfil de segregación escolar por nivel socioeconómico de España. El mismo es el resultado de estimar la magnitud de la segregación para 19 consideraciones de grupo minoritario: desde el 5\% de los estudiantes con familias de menor nivel socioeconómico hasta el $50 \%$ de los estudiantes, y de nuevo hasta el $5 \%$ de los estudiantes de familias con mayor nivel socioeconómico. El resultante es una línea -un perfil-, que se ha representado en un gráfico circular y que aporta información mucho más compleja y útil 
Murillo, F. Javier \& Martínez-Garrido, Cynthia (2019). Perfiles de segregación escolar por nivel socioeconómico en España y sus Comunidades Autónomas. RELIEVE, 25(1), art. 1. doi: http://doi.org/10.7203/relieve.25.1.12917

que la estimación de un único punto, sea el P10, el P20 o Q1.

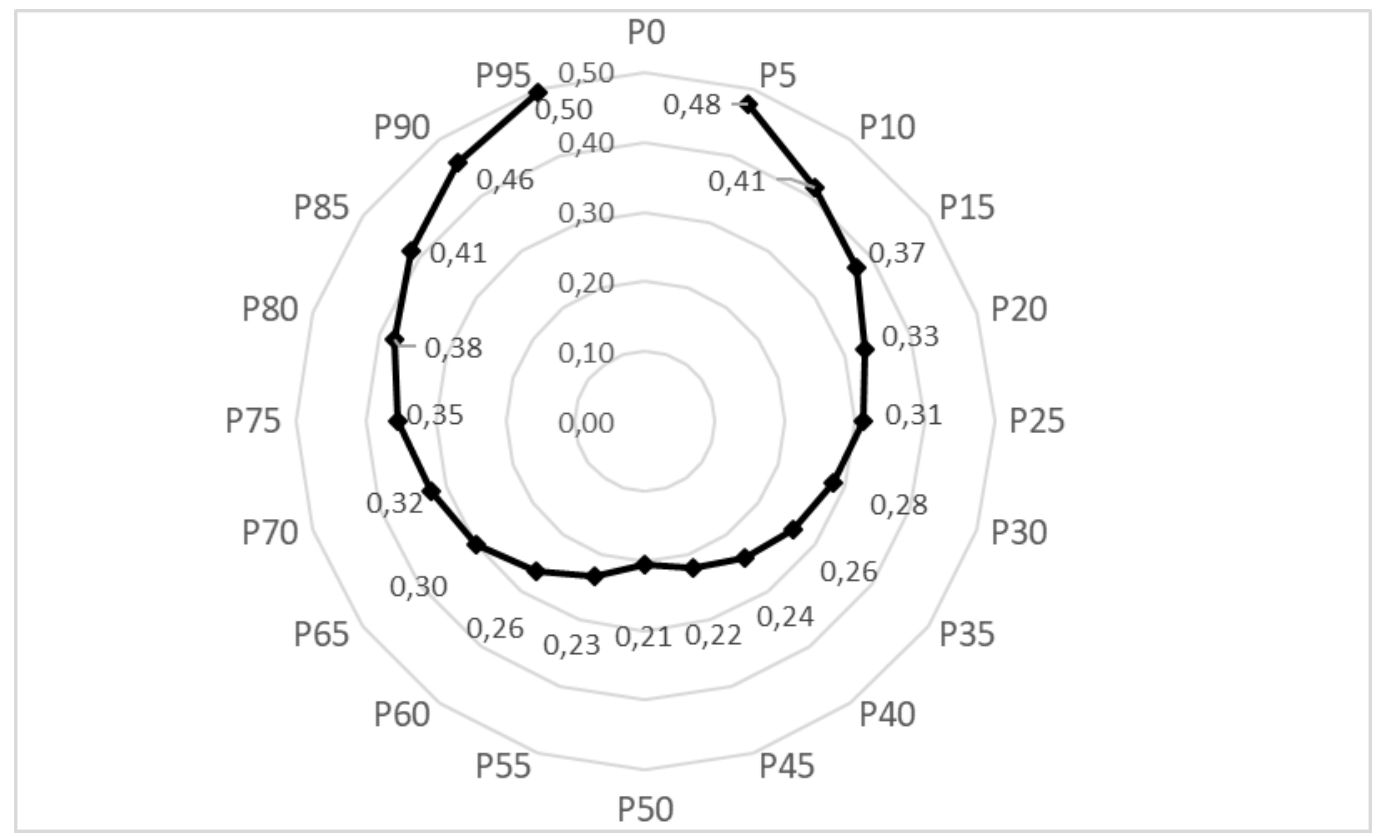

Figura 1. Perfil de segregación escolar por nivel socioeconómico y cultural de España. Índice $\mathrm{G}$ para 19 grupos minoritarios

Fuente: Elaboración propia a partir de PISA 2015.

Del análisis de la figura 1 se pueden extraer algunas ideas. De un lado, la forma de huevo sin cerrar que tiene el perfil, con la parte achatada en el P50 y estirada en el P5 y el P95. Esta representación surge como consecuencia del tamaño del grupo minoritario, un 5\% de sujetos en los extremos superiores y del $50 \%$ en la parte baja. De otro lado, es muy interesante verificar que la segregación en la parte izquierda del perfil es mayor que en la parte derecha. Ello significa que, en el total estatal, la segregación de estudiantes de familias con mayor nivel socioeconómico es superior a la segregación de estudiantes con menor nivel. Esta visión de la segregación ha pasado desapercibida para la práctica totalidad de las investigaciones realizadas hasta el momento. En la tabla A1 del Anexo se presenta toda la información.

Efectivamente, más allá de la verificación de que el punto con menor segregación es el P50 (el valor del índice de Gorard es 0,21), se verifica que la segregación escolar para los grupos de estudiantes con menor nivel socioeconómico es más baja que para los de mayor. Así, para el 10\%, 15\% y $20 \%$ de los estudiantes de familias con menor y mayor nivel socioeconómico las diferencias se maximizan, llegando a los 0,5 puntos entre los puntos de corte. Sin embargo, para el $5 \%$ de los estudiantes las diferencias disminuyen debido al espectacular aumento de la segregación en el P5.

Más interesante, por su carácter comparativo, resulta la estimación del perfil de segregación socioeconómica para cada una de las 17 Comunidades Autónomas de España (figura 2, tabla A1). Sin embargo, el alto número de Comunidades y lo similar de los perfiles hace que la representación gráfica sea poco clara. En todo caso, algunos elementos destacan especialmente: 
Murillo, F. Javier \& Martínez-Garrido, Cynthia (2019). Perfiles de segregación escolar por nivel socioeconómico en España y sus Comunidades Autónomas. RELIEVE, 25(1), art. 1. doi: http://doi.org/10.7203/relieve.25.1.12917

1. El caso de la Comunidad de Madrid es el más llamativo por tener los índices de segregación más altos de todas las regiones durante la mayoría de su recorrido, hasta el P80 en el que es superada por Canarias y hasta llegar al P95 en el que es superada por siete Comunidades. Su segregación promedio en el índice de Gorard es de 0,37. Navarra tiene un perfil parecido, pero más moderado: alto para todos los colectivos, pero bajo para los estudiantes de familias con mayor nivel socioeconómico.

2. Cataluña tiene un comportamiento análogo, tras Madrid es la Comunidad con una mayor segregación para casi todos los puntos de corte. La diferencia es que su segregación se modera en ambos extremos, tanto para los estudiantes de familias con mayor nivel socioeconómico, como para los de menor.

3. En el extremo contrario está Illes Balears, que es la Comunidad con una segregación más baja en prácticamente todos los puntos de corte. Su segregación promedio es de 0,24 , con una varianza baja $(0,07)$.

4. La segregación escolar por nivel socioeconómico en el País Vasco tiene un comportamiento muy característico. Es muy alta para los estudiantes con familias de nivel socioeconómico bajo, pero moderada para los de nivel alto.

5. El Principado de Asturias y Canarias son característicos por su alta variabilidad (var $=0,10)$, por su segregación promedio análoga $(0,31$ y 0,30 respectivamente), pero sobre todo por su comportamiento, ambos con una segregación media para los estudiantes con familias de menor nivel socioeconómico que se incrementa de forma importante para los de mayor nivel.
6. Algo similar a lo que acontece con Extremadura, Castilla y León y Cantabria. Las tres se caracterizan por bajos niveles de segregación para estudiantes con familias de nivel socioeconómico menor, pero aumenta mucho para los de mayor nivel. También con alta variabilidad.

7. La Región de Murcia y la Comunitat Valenciana tienen un comportamiento inverso al anterior: tienen una alta segregación para los percentiles más bajos, y ésta va disminuyendo conforme aumenta el punto de corte del nivel socioeconómico. Ambas, curiosamente con un comportamiento poco estable, aunque más acusado para la Comunitat Valenciana.

8. La Rioja, Andalucía, Galicia y Castilla-La Mancha, con sus diferencias, mantienen comportamientos análogos. Las tres se mantienen en una posición intermedia, sin grandes variaciones para los distintos puntos de corte, con una segregación escolar más baja para La Rioja, media para Andalucía y Galicia, y más alta para Castilla-La Mancha

9. La Rioja, por último, tiene el comportamiento más inestable de todas. Aunque su posición global es intermedia, pasa de ser la tercera Comunidad más segregada en el P25, a ser la menos segregada para P80 y P85.

En todo caso, la tabla 3 refleja la hipótesis de partida de esta investigación: una única cifra, un único grupo minoritario, aporta una imagen poco real de la segregación escolar, en este caso de la Comunidad. Así, según sea ésta, los resultados serán completamente diferentes. 
Murillo, F. Javier \& Martínez-Garrido, Cynthia (2019). Perfiles de segregación escolar por nivel socioeconómico en España y sus Comunidades Autónomas. RELIEVE, 25(1), art. 1. doi: http://doi.org/10.7203/relieve.25.1.12917

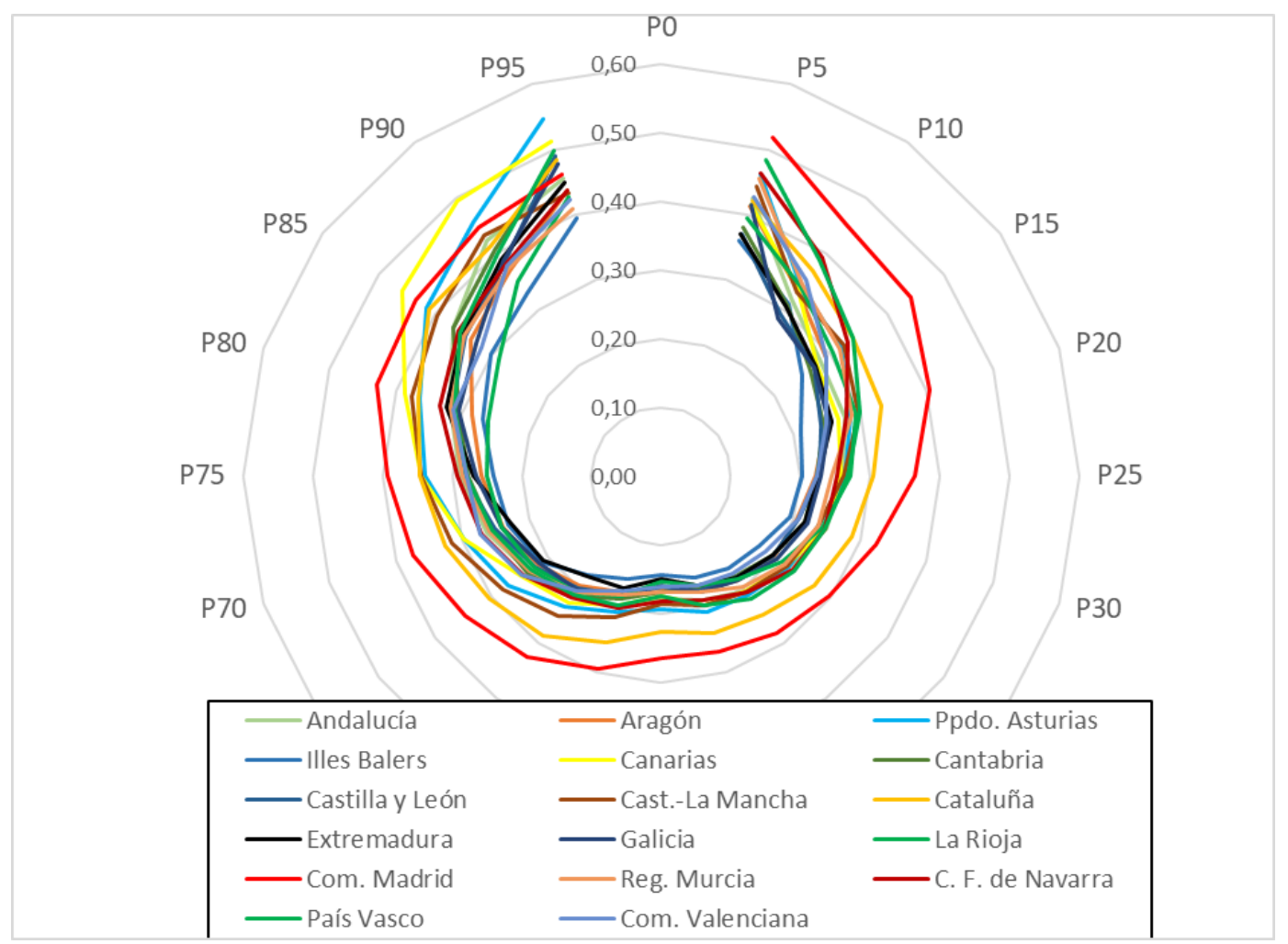

Figura 2. Perfiles de segregación escolar de carácter socioeconómico y cultural en las Comunidades Autónomas de España. Índice G para 19 grupos minoritarios Fuente: Elaboración propia a partir de PISA 2015.

Tabla 3. Orden de las Comunidades Autónomas de España en función de su segregación escolar por nivel socioeconómico

\begin{tabular}{|c|c|c|c|c|c|c|c|c|c|c|c|c|c|c|c|c|c|c|c|}
\hline & P5 & P10 & P15 & P20 & P25 & P30 & P35 & P40 & P45 & P50 & P55 & P60 & P65 & P70 & P75 & P80 & P85 & P90 & P95 \\
\hline les Baleares & IB & GA & IB & IB & IB & IB & IB & IB & IB & 3 & IB & IB & EX & EX & IB & RI & RI & B & IB \\
\hline Extren & EX & $\mathrm{CL}$ & $\mathrm{CA}$ & $\mathrm{CL}$ & AR & AR & VC & VC & VC & EX & EX & EX & IB & IB & RI & IB & IB & RI & MU \\
\hline Castill & $C L$ & EX & CL & CA & CA & C & CA & CA & RI & RI & VC & AR & GA & AR & $\mathrm{R}$ & AR & IC & $\mathrm{R}$ & VC \\
\hline Cantak & CA & $\mathrm{CA}$ & GA & VC & VC & $\mathrm{CL}$ & EX & $\mathrm{CL}$ & EX & GA & AR & $\mathrm{CL}$ & AR & RI & GA & CA & GA & MU & RI \\
\hline La Rioja & RI & IB & EX & AR & EX & CA & $\mathrm{CL}$ & EX & CA & $\mathrm{CL}$ & $\mathrm{CL}$ & GA & $\mathrm{CL}$ & GA & EX & GA & AR & VC & $\mathrm{CM}$ \\
\hline Aragón & AR & AN & $\mathrm{CN}$ & GA & $\mathrm{CL}$ & EX & AR & RI & $\mathrm{CL}$ & VC & MU & VC & RI & $\mathrm{CL}$ & CA & PV & $\mathrm{CL}$ & NA & NA \\
\hline Galicia & GA & CM & AN & EX & GA & GA & GA & AR & AR & AR & GA & RI & PV & PV & VC & VC & MU & $\mathrm{CL}$ & AR \\
\hline Canari & $\mathrm{CN}$ & AR & AR & $\mathrm{CN}$ & MU & AS & RI & GA & MU & MU & RI & $\mathrm{MU}$ & MU & CA & PV & $\mathrm{CL}$ & EX & GA & EX \\
\hline Catalui & CT & $\mathrm{CN}$ & VC & AN & NA & CM & MU & MU & GA & $\mathrm{CA}$ & CA & AN & AN & MU & CL & AN & PV & EX & AN \\
\hline Andalucía & AN & RI & RI & NA & $\mathrm{CN}$ & $\mathrm{MU}$ & $\mathrm{CN}$ & $\mathrm{CN}$ & NA & $\mathrm{CN}$ & $\mathrm{CN}$ & PV & $\mathrm{CA}$ & AN & MU & MU & NA & PV & MD \\
\hline Com. Valencian & VC & AS & MU & AS & AN & RI & CM & AN & AN & PV & PV & CA & NA & NA & AN & EX & AN & CA & GA \\
\hline Cast.-La N & $\mathrm{CM}$ & $\mathrm{MU}$ & AS & MU & $\mathrm{CM}$ & $\mathrm{CN}$ & AS & $\mathrm{CM}$ & PV & AN & AN & NA & VC & VC & NA & NA & $\mathrm{CA}$ & CT & CT \\
\hline Reg.d & MU & VC & CM & RI & AS & NA & AN & NA & CM & NA & NA & $\mathrm{CN}$ & $\mathrm{CN}$ & $\mathrm{CN}$ & AS & AS & CM & AN & CA \\
\hline Ppdo. Asturias & AS & CT & NA & $\mathrm{CM}$ & PV & PV & NA & AS & $\mathrm{CN}$ & $\mathrm{CM}$ & AS & AS & AS & AS & $\mathrm{CM}$ & CT & CT & CM & $\mathrm{CL}$ \\
\hline Navarra & NA & PV & PV & PV & RI & AN & PV & PV & AS & AS & CM & CM & $\mathrm{CM}$ & CM & $\mathrm{CN}$ & CM & AS & MD & PV \\
\hline País Vasco & PV & NA & CT & CT & CT & CT & CT & CT & CT & CT & CT & CT & CT & CT & CT & $\mathrm{CN}$ & MD & AS & $\mathrm{CN}$ \\
\hline Com. Madrid & MD & MD & MD & MD & MD & MD & MD & MD & $\mathrm{MD}$ & MD & MD & MD & MD & MD & MD & MD & $\mathrm{CN}$ & CN & AS \\
\hline
\end{tabular}

Nota: Se ha identificado cada Comunidad Autónoma a través sus iniciales y en un color. Fuente: Elaboración propia a partir de datos de PISA 2015. 
Murillo, F. Javier \& Martínez-Garrido, Cynthia (2019). Perfiles de segregación escolar por nivel socioeconómico en España y sus Comunidades Autónomas. RELIEVE, 25(1), art. 1. doi: http://doi.org/10.7203/relieve.25.1.12917

\section{b) Modelos de segregación}

Aunque para la toma de decisiones la riqueza está en el detalle, 17 perfiles diferentes, uno por cada Comunidad, pueden hacer que se pierda la perspectiva. El análisis de conglomerados permite identificar modelos de perfiles que simplifican la interpretación. De esta forma se ha procedido a hacer un análisis en dos pasos. En primer lugar, un estudio exploratorio mediante un análisis de conglomerados jerárquico que ofrece una imagen global y ayuda a determinar el número de modelos a seleccionar. En segundo término, a través un análisis de K-medias, se analizan las características de los modelos identificados. En ambos casos, se estiman a partir de los 19 grupos minoritarios formados.

Una imagen gráfica de los resultados del análisis de conglomerados jerárquico la aporta el dendograma (figura 3). En el mismo se observa la estructura jerárquica de las Comunidades Autónomas en cuanto a su cercanía según su perfil de segregación escolar por nivel socioeconómico. Lo primero que se observa es que la Comunidad de Madrid tiene un comportamiento completamente diferente al resto de las Comunidades. Parece claro que su situación exige una reflexión.

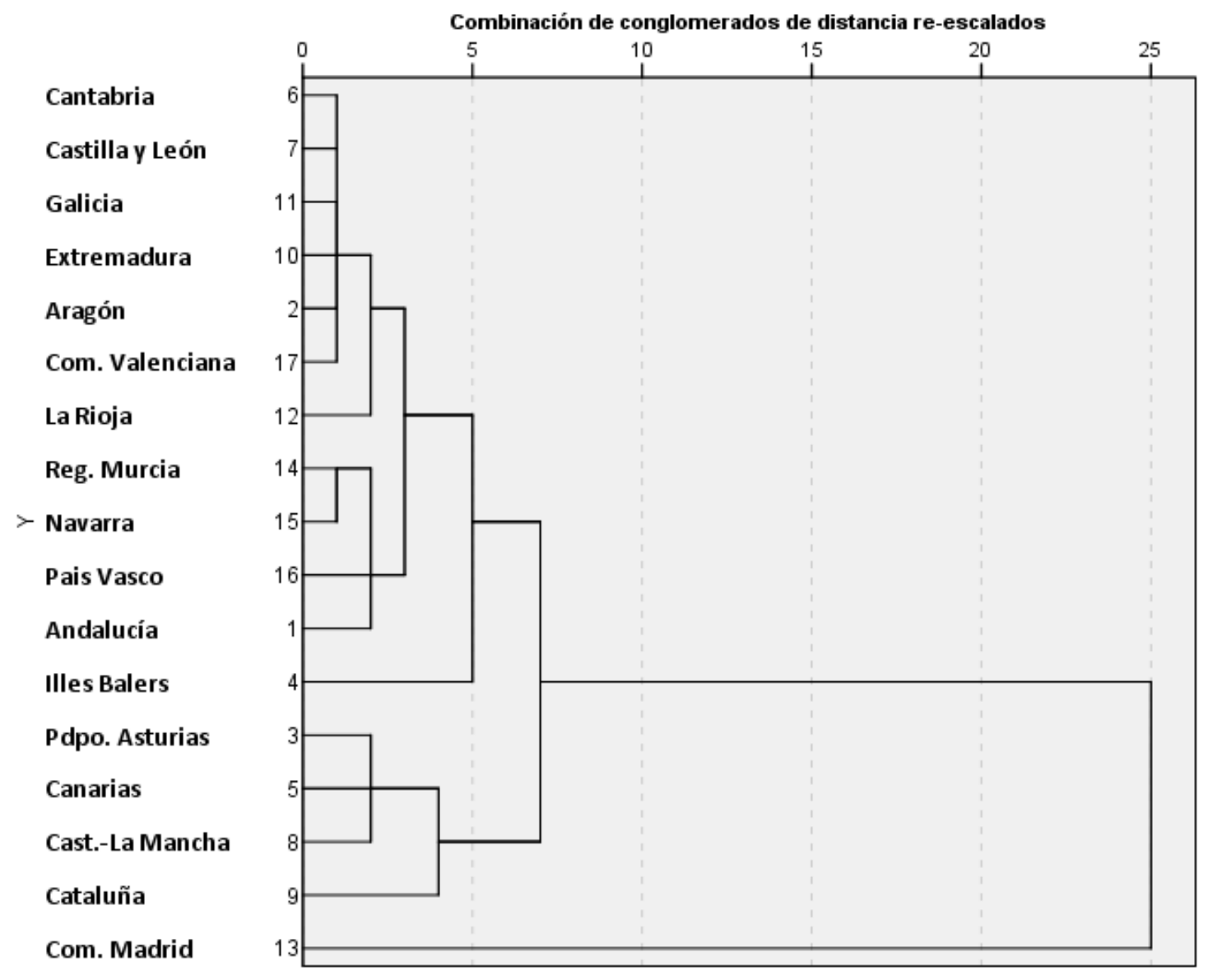

Figura 3. Dendograma con los modelos de segregación escolar de carácter socioeconómico y cultural en las Comunidades Autónomas de España. Resultados del análisis de conglomerados jerárquico con el índice $\mathrm{G}$ para 19 grupos minoritarios

Fuente: Elaboración propia a partir de PISA 2015. 
Murillo, F. Javier \& Martínez-Garrido, Cynthia (2019). Perfiles de segregación escolar por nivel socioeconómico en España y sus Comunidades Autónomas. RELIEVE, 25(1), art. 1. doi: http://doi.org/10.7203/relieve.25.1.12917

El dendograma, además, muestra el comportamiento diferente de Illes Balears, así como la existencia de tres grupos más: el primero conformado por las Comunidades de Cantabria, Castilla y León, Galicia, Extremadura, Aragón, la Comunitat Valenciana y algo más alejado La Rioja; el segundo compuesto por la Región de Murcia, Navarra, País Vasco y Andalucía, y el tercero, en la parte inferior del gráfico, el Principado de Asturias, Canarias, Castilla-La Mancha y Cataluña, esta última algo alejada.

El análisis jerárquico y de K-medias realizado aporta información de las características de estos grupos (tabla A2) y posibilita volver a representar gráficamente los perfiles en un gráfico radial. Esta nueva imagen (figura 4), simplifica la imagen inicial de las Comunidades y aporta una visión más comprensiva que facilita la interpretación de los cinco modelos encontrados:

1. La Comunidad de Madrid, como se ha visto, por si sola representa un modelo. Se caracteriza por tener niveles se segregación muy altos en todos los puntos de corte considerados. Esa tan alta segregación escolar se ve matizada para la segregación del 5\% de estudiantes con familias de mayor nivel socioeconómico y cultural, donde la situación se modera ligeramente.

2. Illes Balears representa el extremo contrario definiéndose por sus bajos niveles de segregación para cualquier grupo minoritario elegido.

3. El tercer grupo, el más numeroso, está conformado por siete Comunidades Autónomas (Andalucía, Aragón, Cantabria, Castilla y León, Extremadura, Galicia y Comunitat Valenciana) y se caracteriza por tener niveles de segregación escolar bajos en general, pero estos son especialmente bajos para los grupos de estudiantes de menos nivel socioeconómico (P5 a P35), que luego se tornan en cifras altas para los grupos definidos por el P90 y P95. Es decir, son Comunidades que presentan una segregación relativamente baja para los estudiantes con familias de menor nivel socioeconómico pero alta para los de mayor.

4. La Región de Murcia, la Comunidad Foral de Navarra, el País Vasco y La Rioja conforman un grupo propio. La forma de su perfil es la más simétrica de los grupos. Se define, así, por tener unos niveles de segregación intermedios y análogos para los estudiantes de mayor y de menor nivel socioeconómico y cultural.

5. Por último, se encuentran las Comunidades de Asturias, Canarias, Castilla-La Mancha y Cataluña. Son Comunidades con una segregación promedio alta, pero con unos niveles de segregación escolar moderados para los niveles bajos de situación socioeconómica y segregación escolar alta para los niveles socioeconómicos más elevados. 
Murillo, F. Javier \& Martínez-Garrido, Cynthia (2019). Perfiles de segregación escolar por nivel socioeconómico en España y sus Comunidades Autónomas. RELIEVE, 25(1), art. 1. doi: http://doi.org/10.7203/relieve.25.1.12917

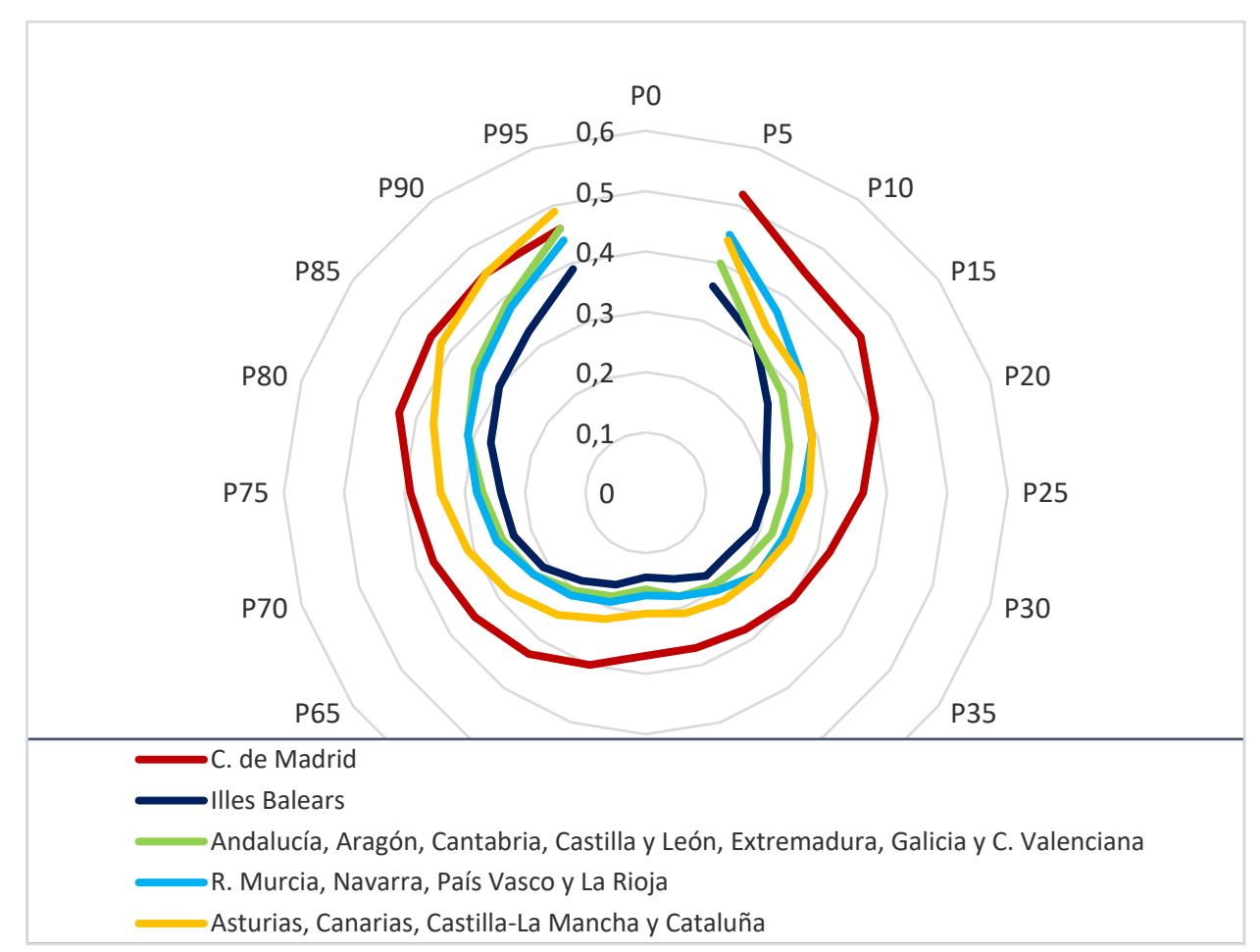

Figura 4. Modelos de segregación escolar de carácter socioeconómico y cultural en las Comunidades Autónomas de España

Fuente: Elaboración propia a partir de PISA 2015.

\section{Conclusiones}

El sistema educativo español tiene un grave problema de segregación social de sus escuelas. Otros estudios (p. ej., Murillo y Martínez-Garrido, 2018a) sitúan a España como uno de los países con una mayor segregación escolar por nivel socioeconómico de Europa, con cifras análogas a los países de Este más segregados y muy alejadas de los países de su contexto más cercano como Francia, Portugal, Alemania o Italia. Además, la variabilidad de situaciones para cada una de las 17 Comunidades Autónomas hace que sea difícil mirarlas como un todo.

Este estudio ha aportado evidencias, en primer lugar, de que estimar la magnitud de la segregación escolar con una sola medición simplifica en exceso una realidad ya de por sí muy compleja. Así, por poner un sencillo ejemplo, Extremadura puede ser la Comunidad con menor segregación escolar por nivel socioeconómico de España o la séptima con mayor segregación, el País Vasco puede ser la segunda Comunidad más segregada o la sexta con menos segregación, todo depende de la consideración del grupo minoritario. Pero no se trata de elegir adecuadamente el punto de corte, cada uno de los grupos minoritarios ofrece una información igualmente relevante para la toma de decisiones.

En segundo término, cumpliendo el objetivo marcado, este trabajo ha determinado el perfil de segregación escolar por nivel socioeconómico en Educación Secundaria Obligatoria en España y sus Comunidades Autónomas. Esta completa imagen aporta mucha información para la toma de decisiones políticas. Por ejemplo, los perfiles de segregación de la Comunidad de Madrid y de Extremadura son opuestos. Para el 5\% de los estudiantes con familias de menos nivel 
Murillo, F. Javier \& Martínez-Garrido, Cynthia (2019). Perfiles de segregación escolar por nivel socioeconómico en España y sus Comunidades Autónomas. RELIEVE, 25(1), art. 1. doi: http://doi.org/10.7203/relieve.25.1.12917

socioeconómico presentan la mayor y la segunda menor segregación de todas las Comunidades, pero para el $5 \%$ de los estudiantes con familias de mayor nivel socioeconómico sus niveles de segregación son muy parecidos. Ello implica que la máxima prioridad de la Comunidad de Madrid es reducir sus niveles de segregación para los estudiantes con familias de menor nivel socioeconómico, mientras que, para la Comunidad de Extremadura, su prioridad debe ser aminorar la segregación para los estudiantes de familias con mayor nivel socioeconómico.

La complejidad de presentar 17 perfiles diferentes justifica la necesidad del segundo objetivo: definir modelos de perfiles. Cumpliendo ese objetivo se han encontrado cinco modelos de comportamiento de la segregación escolar. Su utilidad para la toma de decisiones es, también, clara. Por ejemplo, en aquellas Comunidades donde los resultados muestran que se segrega a los estudiantes con menos recursos es preciso poner en marcha políticas que garanticen que no haya escuelas gueto. Por contra, en las Comunidades donde se segrega a los estudiantes con mayores recursos es fundamental garantizar el cumplimiento de la ley que establece la igualdad de oportunidades de acceso a los estudiantes.

Estos resultados son absolutamente coherentes con los encontrados en estudios que estiman la magnitud de la segregación escolar en España. Así, son coincidentes con los encontrados con Murillo y Martínez-Garrido (2018a), dado que utilizan el mismo índice y la misma base de datos, la diferencia es que en su trabajo sólo aportan 4 grupos minoritarios, frente a los 19 de esta investigación. También son análogos con los de Ferrer, Ferrer y Castel (2006), Mancebón-Torrubia y Pérez-
Ximénez (2010), Ferrer y colaboradores (2011). En estos casos, las diferencias son dos: por una parte, usan el índice de Disimilitud, que genera estimaciones más bajas (Murillo, 2016); y, por otra, que realizan explotaciones especiales de los datos de anteriores ediciones de PISA (2003, 2006 y 2009, respectivamente).

No es el objetivo de este artículo buscar una explicación, ni siquiera tentativa, de las razones que conducen a alcanzar esos niveles de segregación escolar. Sin embargo, la literatura habla, en primer lugar, de factores contextuales tales como de la segregación residencial para explicar la segregación escolar (Denton, 2001; Frankenberg, 2013). De esta forma, la concentración de familias de determinadas características socioeconómicas en barrios justificaría esa concentración en las escuelas. Sin embargo, las grandes diferencias encontradas entre Comunidades llevan a pensar en la importancia de las políticas públicas sobre el tema. Kartsten (2010) habla de dos grandes factores de la política que llevan a una mayor segregación: la política de elección escolar (school choice) y los criterios de admisión de las escuelas privadas.

Analizando a la Comunidad de Madrid, la región en España con una mayor segregación escolar, encontramos que, por una parte, en los últimos años lleva aplicando políticas de elección escolar encaminadas a la creación de un cuasi-mercado escolar tales como el distrito único, por el cual las familias pueden elegir escuela en toda la Comunidad o la publicación de rankings entre centros para facilitar la elección, pero también la política de centros bilingües que segrega a los estudiantes con más dificultades de aquellos que no lo son. $\mathrm{Y}$, por otra parte, es tras el País Vasco, la Comunidad con mayor porcentaje de 
Murillo, F. Javier \& Martínez-Garrido, Cynthia (2019). Perfiles de segregación escolar por nivel socioeconómico en España y sus Comunidades Autónomas. RELIEVE, 25(1), art. 1. doi: http://doi.org/10.7203/relieve.25.1.12917

estudiantes matriculados en escuelas públicas. Por cierto, el País Vasco es una de las Comunidades con una mayor segregación para los estudiantes con familias de menor nivel socioeconómico. Sin duda hacen falta más investigaciones que ayuden a comprender estos fenómenos.

En este estudio se ha trabajado con datos de PISA 2015. Este hecho le otorga su mayor fortaleza, pero también algunas debilidades. Entre las primeras hay que destacar la posibilidad de contar con una muestra estadísticamente representativa de estudiantes de las 17 Comunidades Autónomas y con una medida del nivel socioeconómico y cultural tan fiable. Jamás hasta este momento la comunidad científica ha contado con datos de tal alta calidad y fiables para hacer un estudio de este tipo. Sin embargo, también hay que señalar alguna limitación, como es contar con tan pocos estudiantes por escuela, lo que limita la fiabilidad para grupos minoritarios muy pequeños. Debilidades, en todo caso, pequeñas en comparación con las fortalezas y que generan sólidas evidencias empíricas.

Esta investigación abre un camino que es necesario seguir y profundizar. Vista la diferente segregación de cada Comunidad para cada grupo minoritario resulta necesario profundizar en las causas y las consecuencias de esta segregación escolar, especialmente para los grupos de estudiantes con familias con mayor nivel socioeconómico y cultural, que ha sido mucho menos estudiada. Así como analizar las políticas educativas que han llevado a esta situación y las que pueden revertirla.

La segregación escolar atenta gravemente contra la igualdad de oportunidades educativas de todos los estudiantes, impidiendo de manera decisiva la construcción de una sociedad más justa e inclusiva. Este artículo ha demostrado lo poco que se sabe sobre la segregación en España. Ahora el reto es tomar las medidas para reducirla drásticamente. La sociedad no puede esperar.

\section{Referencias}

Arenas, A. \& Hindriks, J. (2017). Intergenerational mobility, school inequality and social segregation. Lovaina: Université Catholique de Louvain.

Bell, W. (1954). A probability model for the measurement of ecological segregation. Social Forces, 32, 357364. https://doi.org/10.2307/2574118

Benavides, M., León, J., \& Etesse, M. (2014). Desigualdades educativas y segregación en el sistema educativo peruano. Una mirada comparativa de las pruebas PISA 2000 y 2009. Lima: GRADE.

Benito, R. \& González, I. (2007). Processos de segregació escolar a Catalunya. Barcelona: Mediterrània.

Bonal, X. (2018). La política educativa ante el reto de la segregación escolar en Cataluña. París: UNESCO.

Coleman, J., Hoffer, T., \& Kilgore, S. (1982). High school achievement. Public, catholic and private schools compared. Nueva York, NY: Basic Books.

Denton, N. (2001). The persistence of segregation: Links between residential segregation and school segregation. En J. Powell, G. Kearney y V. Kay (Eds.), In pursuit of a dream deferred: Linking housing and education policy (pp. 89-119). Nueva York, NY: Peter Lang.

Duncan, O. \& Duncan, B. A. (1954). Methodological analysis of segregation indexes. American Sociological Review, 20(2), 210-217. https://doi.org/10.2307/2088328 
Murillo, F. Javier \& Martínez-Garrido, Cynthia (2019). Perfiles de segregación escolar por nivel socioeconómico en España y sus Comunidades Autónomas. RELIEVE, 25(1), art. 1. doi: http://doi.org/10.7203/relieve.25.1.12917

Ferrer, F., Castejón, A., Castel, J. L., \& Zancajo, A. (2011). PISA 2009: Evaluació de les desigualtats educatives a Catalunya. Barcelona: Fundació Jaume Bofill.

Ferrer, F., Ferrer, G., \& Castel, J. L. (2006). Les desigualtats educatives a Catalunya: PISA 2003. Barcelona: Fundació Jaume Bofill.

Ferrer, F., Ferrer, G., \& Castel, J. L. (2008). Les desigualtats educatives a Catalunya (I-II). Barcelona: Fundació Jaume Bofill.

Frankenberg, E. (2013). The role of residential segregation in contemporary school segregation. Education and Urban Society, 45(5), 548-570.

https://doi.org/10.1177/001312451348 $\underline{6288}$

Gasparini, L., Jaume, D., Serio, M., \& Vázquez, E. (2011). La segregación entre escuelas públicas y privadas en Argentina. Reconstruyendo la evidencia. Desarrollo Económico, 51(203), 189-219.

Gorard, S. (2006). What does an index of school segregation measure? A commentary on Allen and Vignoles. York: Department of Educational Studies.

Gorard, S. \& Taylor, C. (2002). What is segregation? A comparison of measures in terms of 'strong' and 'weak' compositional invariance. Sociology, 36(4), 875-895. https://doi.org/10.1177/003803850203 $\underline{600405}$

Hutchens, R. (2004). One measure of segregation. International Economic Review, 45(2), 555-578. https://doi.org/10.1111/j.14682354.2004.00136.X

Jahn, J., Schmid, C., \& Schrag, C. (1947). The measurement of ecological segregation. American
Sociological Review, 12, 293-303. http://dx.doi.org/10.2307/2086519

Jaume, D. (2013). Un estudio sobre el incremento de la segregación escolar en Argentina. La Plata: CEDLAS.

Kartsten, S. (2010). School segregation. En OECD. (Ed.), Equal opportunities? The labor market integration of the children of immigrants (pp. 193-210). París: OECD.

Krüguer, N. (2014). Más allá del acceso: Segregación social e inequidad en el sistema educativo. Cuadernos de Economía, 33(63), 513-542. https://doi.org/10.15446/cuad.econ.v3 3n63.45344

Mancebón-Torrubia, M. J. \& PérezXiménez, D. (2009). Segregación escolar en el sistema educativo español. Un análisis a partir de PISA 2006. En J. Cordero y R. Simancas (Eds.), Investigaciones de economía de la educación (pp. 63-75). Valencia: AEDE.

Mancebón-Torrubia, M. J. \& PérezXiménez, D. (2010). Una valoración del grado de segregación socioeconómica existente en el sistema educativo español. Un análisis por Comunidades Autónomas a partir de PISA 2006. Regional and Sectoral Economic Studies, 10(3), 129-148.

Mancebón-Torrubia, M. J. \& PérezXiménez, D. (2014). Equality of school choice: A study applied to the Spanish region of Aragón. Education Economics, 22(1), 90-111. https://doi.org/10.1080/09645292.201 $\underline{0.545197}$

Martínez, L. \& Ferrer, A. (2018). Mézclate conmigo. De la segregación socioeconómica a la educación inclusiva. Madrid: Save the Children España.

Massey, D. S., Rothwell, J., \& Domina, T. (2009). The changing bases of segregation in the United States. The 
Murillo, F. Javier \& Martínez-Garrido, Cynthia (2019). Perfiles de segregación escolar por nivel socioeconómico en España y sus Comunidades Autónomas. RELIEVE, 25(1), art. 1. doi: http://doi.org/10.7203/relieve.25.1.12917

Annals of the American Academy of Political and Social Science, 626(1), 74-90.

https://doi.org/10.1177/000271620934 $\underline{3558}$

Murillo, F. J. (2016). Midiendo la segregación escolar en América Latina. Un análisis metodológico utilizando el TERCE. REICE. Revista Iberoamericana sobre Calidad, Eficacia y Cambio en Educación, 14(4), 33-60. https://doi.org/10.15366/reice2016.14. 4.002

Murillo, F. J. \& Martínez-Garrido, C. (2017). Estimación de la magnitud de la segregación escolar en América Latina. Magis, Revista Internacional de Investigación en Educación, 9(19), 11-30.

https://doi.org/10.11144/javeriana.m919.emse

Murillo, F. J. \& Martínez-Garrido, C. (2018a). Magnitud de la segregación escolar por nivel socioeconómico en España y sus comunidades autónomas y comparación con los países de la Unión Europea. RASE: Revista de Sociología de la Educación, 11(1), 3758.

https://doi.org/10.7203/RASE.11.1.10 $\underline{129}$

Murillo, F. J. \& Martínez-Garrido, C. (2018b). Incidencia de la crisis económica en la segregación escolar en España / Impact of the economic crisis on school segregation in Spain. Revista de Educación, 381, 67-93. https://doi.org/10.4438/1988-592XRE-2017-381-381
OCDE. (2010). PISA 2009 results: Overcoming social background. Equity in learning opportunities and outcomes. París: OCDE.

OCDE. (2016). PISA 2015 Assessment and analytical framework. París: OCDE.

https://doi.org/10.1787/978926425542 5-en

Sánchez Hugalde, A. (2007). La segregació escolar dels immigrants a Catalunya. Quaderns D'avaluació, 13, 55-76.

Sicilia, G. \& Simancas, R. (2018). Equidad educativa en España: Comparación regional a partir de PISA 2015. Madrid. Fundación Ramón Areces

Síndic. (2008). La segregación escolar en Cataluña. Barcelona: Síndic.

St. John, N. H. (1966). The effect of segregation on the aspirations of negro youth. Harvard Educational Review, 36(3), 284-294. https://doi.org/10.17763/haer.36.3.137 368j68m5u9800

Valenzuela, J. P., Bellei, C., \& De Los Ríos, D. (2008). Evolución de la segregación socioeconómica de los estudiantes chilenos y su relación con el financiamiento compartido. Santiago de Chile: FONIDE, Ministerio de Educación.

Vázquez, E. (2012). Segregación escolar por nivel socioeconómico. Midiendo el fenómeno y explorando sus determinantes. Buenos Aires: CEDLAS.

\section{Agradecimientos}

Este artículo ha sido desarrollado en el marco del Proyecto I+D+i de Excelencia "Escuelas en contextos socio-económicamente desafiantes: Una aproximación desde la Educación para la Justicia Social de Investigación” Ref: EDU2014-56118-P. Financiado con fondos del Ministerio de Economía y Competitividad-Gobierno de España. 


\section{$\underline{\text { Anexos }}$}

Tabla A1. Magnitud de la segregación escolar por nivel socioeconómica en España a partir de la consideración de diferentes criterios de consideración del grupo minoritario en percentiles del nivel socioeconómico y cultural de las familias de los estudiantes

\begin{tabular}{|c|c|c|c|c|c|c|c|c|c|c|c|c|c|c|c|c|c|c|c|}
\hline & P5 & 10 & 15 & 20 & 25 & 30 & 35 & 40 & 15 & P50 & 5 & P60 & 5 & P70 & 875 & 880 & & & \\
\hline & & & & & & & & & & & & & & & & & & & \\
\hline & & & & & & & & & & & & & & & & & & & \\
\hline & & & & & & & & & & & & & & & & & & & \\
\hline & & & & & & & & & & & & & & & & & & & \\
\hline & & & & & & & & & & & & & & & & & & & \\
\hline & & & & & & & & & & & & & & & & & & & \\
\hline & & & & & & & & & & & & & & & & & & & \\
\hline & & & 46 & 967 & & & & & & & & & & & & & & & 4323 \\
\hline & & & & & & & & & & & & & & & & & & & 483 \\
\hline & & & & & & & & & & & & & & & & & & & 448 \\
\hline & & & & & & & & & & & & & & & & & & & , 477€ \\
\hline & & & & & & & & & & & & & & & & & & & 4277 \\
\hline & & 517 & & & & & & & & & & & & & & & & & 4615 \\
\hline & & 3443 & 3159 & 2866 & 2439 & 2372 & 2186 & & 0,1767 & 1689 & 1814 & 0,2095 & 2337 & 0,2593 & 0,2844 & & 0,3488 & 727 & 0,4096 \\
\hline varra & 631 & 3919 & 3304 & 2799 & 2526 & 2478 & 2329 & 2091 & 0,1902 & 1829 & 0,2018 & 2193 & 2439 & 0,2720 & 0,2931 & 3339 & ,3597 & & 436 \\
\hline & & 3879 & 3402 & 2987 & 2678 & 2478 & 2348 & 2198 & 1969 & 1745 & 0,1986 & & 2310 & 2543 & ,2794 & & 3556 & 3991 & , 497؟ \\
\hline & & 3521 & 2927 & 2484 & 2234 & 2061 & , 1858 & 750 & 670 & 616 & 0,1755 & 063 & 2461 & 731 & 0,2793 & 129 & ,3175 & 0,3776 & 0,4235 \\
\hline spaña & 0,4781 & 0,4145 & 0,3730 & 0,3318 & 0,3114 & 0,2832 & 0,2622 & 0,2433 & 0,2202 & 0,2060 & 0,2347 & 0,2646 & 0,3005 & 0,3230 & 0,354 & 0,3770 & 0,414 & 0,4583 & 49 \\
\hline
\end{tabular}

Fuente: Elaboración propia a partir de datos de PISA 2015. 
Tabla A2. Modelos de segregación escolar de carácter socioeconómico y cultural en las Comunidades Autónomas de España. Centros de conglomerados finales del análisis de conglomerados de K-medias

\begin{tabular}{|c|c|c|c|c|c|}
\hline & C. de Madrid & Illes Balears & $\begin{array}{l}\text { Andalucía, Aragón, } \\
\text { Cantabria, Castilla y } \\
\text { León, Extremadura, } \\
\text { Galicia y Com. } \\
\text { Valenciana }\end{array}$ & $\begin{array}{l}\text { Reg. Murcia, Navarra, } \\
\text { País Vasco y La Rioja }\end{array}$ & $\begin{array}{c}\text { Ppdo. Asturias, } \\
\text { Canarias, Castilla-La } \\
\text { Mancha y Cataluña }\end{array}$ \\
\hline $\mathrm{P5}$ & 0,52 & 0,36 & 0,40 & 0,45 & 0,44 \\
\hline P10 & 0,45 & 0,31 & 0,31 & 0,37 & 0,34 \\
\hline P15 & 0,44 & 0,25 & 0,28 & 0,32 & 0,32 \\
\hline P20 & 0,40 & 0,21 & 0,25 & 0,29 & 0,29 \\
\hline P25 & 0,36 & 0,20 & 0,23 & 0,26 & 0,27 \\
\hline P30 & 0,32 & 0,19 & 0,22 & 0,24 & 0,25 \\
\hline P35 & 0,30 & 0,17 & 0,20 & 0,23 & 0,23 \\
\hline P40 & 0,28 & 0,17 & 0,19 & 0,20 & 0,22 \\
\hline P45 & 0,27 & 0,15 & 0,18 & 0,18 & 0,21 \\
\hline P50 & 0,27 & 0,14 & 0,16 & 0,17 & 0,20 \\
\hline P55 & 0,30 & 0,16 & 0,18 & 0,19 & 0,22 \\
\hline P60 & 0,33 & 0,18 & 0,20 & 0,21 & 0,25 \\
\hline P65 & 0,35 & 0,21 & 0,23 & 0,23 & 0,28 \\
\hline P70 & 0,37 & 0,23 & 0,25 & 0,26 & 0,31 \\
\hline P75 & 0,39 & 0,24 & 0,27 & 0,28 & 0,34 \\
\hline P80 & 0,43 & 0,27 & 0,31 & 0,31 & 0,37 \\
\hline P85 & 0,44 & 0,30 & 0,35 & 0,34 & 0,42 \\
\hline P90 & 0,45 & 0,33 & 0,39 & 0,38 & 0,45 \\
\hline P95 & 0,46 & 0,39 & 0,46 & 0,44 & 0,49 \\
\hline
\end{tabular}

Fuente: Elaboración propia a partir de datos de PISA 2015.

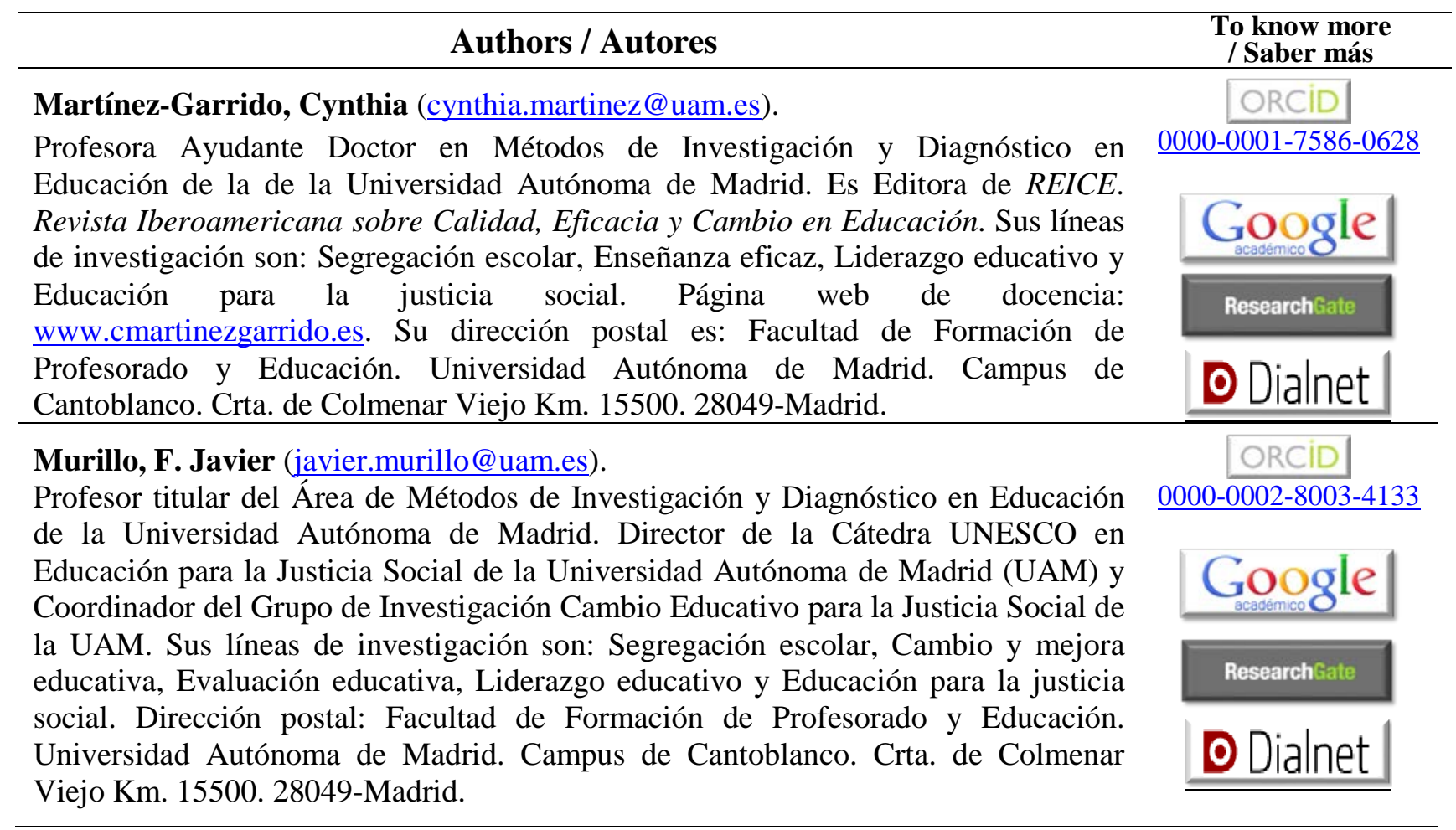




\section{RELIEVE}

Revista ELectrónica de Investigación y $\mathbf{E V}$ aluación Educativa E-Journal of Educational Research, Assessment and Evaluation

[ISSN: 1134-4032]

\section{(c) $(1)(9)$}

Esta obra tiene licencia de Creative Commons Reconocimiento-NoComercial 4.0 Internacional. This work is under a Creative Commons Attribution 4.0 International license. 